\title{
Identification of yeasts by RFLP analysis of the 5.85 rRNA gene and the two ribosomal internal transcribed spacers
}

\author{
B. Esteve-Zarzoso, ${ }^{1,2}$ C. Belloch, ${ }^{1}$ F. Uruburu ${ }^{1}$ and A. Querol ${ }^{2}$
}

Author for correspondence: A. Querol. Tel: +34639000 22. Fax: +34 63636301 . e-mail: aquerol@iata.csic.es

\author{
1 CECT (Spanish Type \\ Culture Collection), \\ Universitat de València, \\ Edificio de Investigación, \\ Campus de Burjassot, \\ 46100 Burjassot, València, \\ Spain \\ 2 Departamento de \\ Biotecnología, Instituto de \\ Agroquímica y Tecnología \\ de Alimentos (CSIC), P. Box \\ 73, 46100 Burjassot, \\ València, Spain
}

\begin{abstract}
The identification and classification of yeasts have traditionally been based on morphological, physiological and biochemical traits. Various kits have been developed as rapid systems for yeast identification, but mostly for clinical diagnosis. In recent years, different molecular biology techniques have been developed for yeast identification, but there is no available database to identify a large number of species. In the present study, the restriction patterns generated from the region spanning the internal transcribed spacers (ITS1 and ITS2) and the 5.8S rRNA gene were used to identify a total of 132 yeast species belonging to $\mathbf{2 5}$ different genera, including teleomorphic and anamorphic ascomycetous and basidiomycetous yeasts. In many cases, the size of the PCR products and the restriction patterns obtained with endonucleases Cfol, Haell and Hinfl yielded a unique profile for each species. Accordingly, the use of this molecular approach is proposed as a new rapid and easy method of routine yeast identification.
\end{abstract}

Keywords: yeast identification, RFLP, 5.8S-ITS region

\section{INTRODUCTION}

The beneficial activities of yeasts are of great economic significance and they have been used for millennia in the production of fermented foods and alcoholic beverages (Deák \& Beuchat, 1996). In the present century, as the fermentation industry has developed, yeasts have been used in the production of valuable products, including antibiotics, vitamins and enzymes. However, they also cause immense economic losses, and their harmful activities as saprotrophs include damage to food and manufactured goods. Consequently, the reliable and rapid identification of those species involved in food processing and the detection of yeasts causing food spoilage are desirable (Carlie \& Watkinson, 1994).

Yeasts are unicellular fungi and can be classified into two phylogenetic groups, teleomorphic and anamorphic ascomycetous yeasts and teleomorphic and anamorphic basidiomycetous yeasts (Kurtzman \& Fell, 1998). The differentiation of taxa is usually

Abbreviation: ITS, internal transcribed spacer. achieved by comparison of morphological traits and physiological features (Barnett et al., 1990; Krejer-van $\mathrm{Rij}, 1984)$, which in some cases can lead to an incorrect classification of species or a false identification of strains.

The conventional methodology for yeast identification requires evaluation of some 60-90 tests, and the process is complex, laborious and time consuming (Deák, 1995; Deák \& Beuchat, 1996). In recent years, to improve the conventional methods, rapid kits for yeast identification have been developed. However, they were initially designed for clinical diagnosis and their application is restricted to $40-60$ yeast species of medical interest (Deák, 1993). In general, it is necessary to do around 100 tests to obtain a reliable identification of yeasts at the species level, and 1-3 weeks are often necessary to obtain a final result (Barnett et al., 1990; Krejer-van Rij, 1984; Lin \& Fung, 1987). Some methods based on the analysis of total cell proteins (Guillamón et al., 1993; van Vuuren \& van Der Meer, 1987; Vacanneyt et al., 1991) and long-chain fatty acids using gas chromatography (Augustyn et al., 1991; Cottrell et al., 1986; Moreira da Silva et al., 1994; Tredoux et al., 1987) have also been developed. However, the reproducibility of these techniques is 
questionable due to the fact that they depend on the physiological state of the yeast cells (Golden et al., 1994).

Recent progress in molecular biology has led to the development of new techniques for yeast identification based on similarity or dissimilarity of DNA, RNA or proteins. These include allozyme patterns (Naumov et al., 1997), DNA-DNA hybridization (Török et al., 1993; Vaughan Martini \& Martini, 1985, 1987), electrophoretic karyotyping (Guillamón et al., 1996; Nadal et al., 1996; Perez et al., 1995, Querol et al., 1992b, Schüctz \& Gafner, 1993; Török et al., 1993), microsatellite analysis (Baleiras Couto et al., 1996), nested-PCR (Ibeas et al., 1996), random amplified polymorphic DNA (RAPD) analysis (Baleiras Couto et al., 1994; Lopandic et al., 1996; Quesada \& Cenis, 1995), RFLP of chromosomal DNA (Versavaud \& Hallet, 1995) or RFLP of mitochondrial DNA (Belloch et al., 1997; Guillamón et al., 1994, 1997; Ibeas et al., 1997; Nadal et al., 1996; Pérez et al., 1995; Querol et al., 1992a, Romano et al., 1996). However, these techniques are impractical for the routine identification of a large number of species since they were developed for species characterization and there is no available database which would permit the analysis of new results.

Previous results have demonstrated that the complex ITS (internal transcribed spacer) regions (non-coding and variable) and the 5.8S rRNA gene (coding and conserved) are useful in measuring close fungus genealogical relationships since they exhibit far greater interspecific differences than the $18 \mathrm{~S}$ and $25 \mathrm{~S}$ rRNA genes (Cai et al., 1996; James et al., 1996; Kurtzman, 1992, 1993). Because ribosomal regions evolve in a concerted fashion, they show a low intraspecific polymorphism, and a high interspecific variability $(\mathrm{Li}$, 1997) has been proved very useful for the classification of Saccharomyces species (Huffman et al., 1992; Molina et al., 1992; Valente et al., 1996; Wyder \& Puhan, 1997), Kluyveromyces species (Belloch et al., 1998) and, recently, for the identification of a small collection of wine yeast species (Guillamón et al., 1998).

In the present study, we have tested the application of the restriction analysis of the rRNA region spanning the 5.8 rRNA gene and the two ITSs (here referred to as the 5.8S-ITS region) as a rapid and easy method for yeast species identification. For this purpose, we have obtained restriction patterns of PCR products for the identification of 132 species from 25 different genera, including teleomorphic and anamorphic ascomycetous and basidiomycetous yeasts.

\section{METHODS}

Yeast strains. We have analysed 243 different strains belonging to 132 different yeast species of 25 genera, obtained from the Spanish Type Culture Collection (CECT). To keep this study to a manageable size, we have mainly relied on type strains representing the 132 yeast species most frequently isolated from food. For some species, when substantial heterogeneity was suspected, or due to their biotechnological importance, additional strains were also characterized. The designations of the strains are listed in Tables 1-3.

PCR reaction and DNA digestions. Cells were directly collected from a fresh yeast colony using a yellow tip and suspended in $100 \mu \mathrm{l}$ PCR reaction mix containing $0.5 \mu \mathrm{M}$ primer ITS1 (5' TCCGTAGGTGAACCTGCGG 3'), $0 \cdot 5 \mu \mathrm{M}$ primer ITS4 $\left(5^{\prime}\right.$ TCCTCCGCTTATTGATATGC $\left.3^{\prime}\right), 10 \mu \mathrm{M}$ deoxynucleotides, $1.5 \mathrm{mM} \mathrm{MgCl}_{2}$ and $1 \times$ buffer (MAD-GEN). The suspension was heated at $95^{\circ} \mathrm{C}$ for $15 \mathrm{~min}$ in a Progene (Techne) thermocycler. One unit of DNA Polymerase SuperTherm (MAD-GEN) was then added to each tube. PCR conditions were as follows: initial denaturation at $95^{\circ} \mathrm{C}$ for $5 \mathrm{~min} ; 35$ cycles of denaturing at $94{ }^{\circ} \mathrm{C}$ for $1 \mathrm{~min}$, annealing at $55.5^{\circ} \mathrm{C}$ for $2 \mathrm{~min}$ and extension at $72^{\circ} \mathrm{C}$ for $2 \mathrm{~min}$; and a final extension at $72{ }^{\circ} \mathrm{C}$ for $10 \mathrm{~min}$. PCR products $(10 \mu \mathrm{l}$ or approximately $0.5-1.0 \mu \mathrm{g})$ were digested without further purification with the restriction endonucleases CfoI, HaeIII and HinfI (Boehringer Mannheim), although endonucleases $A l u \mathrm{I}, D d e \mathrm{I}, S c \mathrm{FFI}$ or TaqI were additionally used in some particular cases. The PCR products and their restriction fragments were separated on $1.4 \%$ and $3 \%$ agarose gels, respectively, with $1 \times \mathrm{TAE}$ buffer. After electrophoresis, gels were stained with ethidium bromide, visualized under UV light and photographed (Image Master, Pharmacia). Sizes were estimated by comparison against a DNA length standard (100 bp ladder, Gibco-BRL).

\section{RESULTS AND DISCUSSION}

ITS1 and ITS4 primers were used to amplify the region of the rDNA repeat unit that includes the 5.8S rRNA gene and the two non-coding regions designated the internal transcribed spacers (ITS1 and ITS2) (White et al., 1990) of 243 strains belonging to 132 species. Tables 1, 2 and 3 show the sizes of the PCR products and the restriction fragments obtained using the restriction endonucleases CfoI, HaeIII and HinfI. Fragments smaller than $50 \mathrm{bp}$ could not be reproducibly visualized and were not included in these tables.

The PCR products showed a high degree of length variation, ranging from $380 \mathrm{bp}$ for Yarrowia lipolytica (CECT 1240) and Pichia pastoris (CECT 11078) to $1050 \mathrm{bp}$ for the type strain of Schizosaccharomyces pombe var. pombe. In the majority of cases, the PCR products from strains of the same species had identical molecular sizes, and species of the same genus had similar sizes for the amplified fragment. In the following sections results are discussed for each yeast class, genus by genus.

\section{Teleomorphic and anamorphic ascomycetous yeasts}

The genus Candida includes all yeast species that cannot be classified in other asexual ascomycetous yeast genera. As a result it is a very heterogeneous genus and the perfect state of most Candida species is 
still unknown (Krejer-van Rij, 1984). Different molecular techniques have been used to identify and characterize Candida species. However, except in the case of the clinical species (Botelho \& Planta, 1994; Iwaguchi et al., 1990; Jordan, 1994), a standard method of classification has not been developed. In this study, we have analysed by RFLP of the 5.8S-ITS region 56 strains belonging to 30 species. These strains showed a unique restriction pattern for each species with the three endonucleases used (see Table 1). The size of the amplified fragment was very variable, ranging from $400 \mathrm{bp}$ for Candida agrestis (CECT $11179^{\mathrm{T}}$ ) to $800 \mathrm{bp}$ for Candida glabrata (CECT 1448), showing the heterogeneity of this genus. Due to the high diversity and complexity of this genus we recommend use of the information obtained with the three restriction enzymes CfoI, HaeIII and HinfI to achieve a reliable classification of the 31 species included in this study.

Debaryomyces is a complex genus in which species previously identified as Saccharomyces, Torulaspora, Zygosaccharomyces, Issatchenkia and Pichia have been included. In our study, three species, Debaromyces hansenii, Debaromyces polymorphus and Debaromyces pseudopolymorphus, were analysed. Because some of the morphological and physiological tests produce variable or delayed responses, it is difficult to achieve a correct identification of the species of this genus by classical methods (Barnett et al., 1990; Krejer-van Rij, 1984). In most cases, restriction analysis of the 5.8S-ITS region of Debaryomyces species exhibited the same patterns with different endonucleases, not only with the three general restriction enzymes included in Table 1, but also with $A l u \mathrm{I}, D d e \mathrm{I}$, ScrFI and TaqI (results not shown). Some strains of Debaromyces polymorphus showed a different pattern, indicating that this taxon includes two different types of strains. One type had the same pattern as the other two species of the genus. The second type exhibited a clearly different pattern, and therefore their assignation to this species could be reconsidered in further studies. In the light of these results and due to the heterogeneity of this genus, more strains representing all the species from this genus should be used to verify our results.

As representatives of the genus Dekkera/ Brettanomyces, six strains of Dekkera anomala and Dekkera bruxellensis were analysed. Both species are important spoilage yeasts present in soft drinks and alcoholic beverages. The restriction patterns obtained with the three restriction enzymes are species-specific. A striking result is the large difference in the PCR product length, namely 800 bp for Dekkera anomala, which is in the range of the largest fragments, and $485 \mathrm{bp}$ for Dekkera bruxellensis, in the range of the shortest sizes. Another very interesting result is the fact that both anamorph (Brettanomyces bruxellensis, CECT $1009^{\mathrm{LT}}$ ) and teleomorph forms (Dekkera bruxellensis, CECT $1451^{\mathrm{T}}$ ) exhibited the same PCR lengths and restriction patterns, indicating that this technique is also useful to detect perfect-imperfect pairs.

Only six species are currently accepted in the genus Hanseniaspora (anamorph $=$ Kloeckera) according to DNA reassociation studies (Meyer et al., 1978) and partial 18 S and 26S rRNA nucleotide sequence analysis (Yamada et al., 1992). According to our results, Hanseniaspora guilliermondii and Hanseniaspora uvarum are closely related species, both showing the same pattern using AluI, CfoI, HaeIII, HinfI, ScrFI and $\operatorname{Taq} \mathrm{I}$ restriction endonucleases. However, their differentiation can be obtained with the use of DdeI (Table 2). For the remaining species the use of only one enzyme (CfoI, HaeIII or HinfI) is sufficient to obtain a pattern representative of each species.

In the genus Kluyveromyces, the approximate lengths of the amplified products were enough to identify the majority of the species. However, to differentiate among the species Kluyveromyces aestuarii, Kluyveromyces dobzhanskii, Kluyveromyces lactis, Kluyveromyces marxianus and Kluyveromyces wickerhamii, the digestion of the PCR product with restriction enzymes HaeIII and HinfI was required. The pair Kluyveromyces thermotolerans and Kluyveromyces waltii presented the same pattern with all the restriction enzymes used. All the strains belonging to the same species showed the same pattern with the different endonucleases, with the exception of the strains of Kluyveromyces lactis formerly classified as Kluyveromyces marxianus var. drosophilarum (CECT 10390 and 11337) and Kluyveromyces marxianus var. phaseolosporus (CECT 11340), which exhibited different patterns with HinfI.

According to morphological and physiological tests, ten species are recognized in the genus Metschnikowia. Four of them have been isolated from aquatic sources and the others from terrestrial environments. Metschnikowia pulcherrima is one of the most important species involved in food processing. We analysed eight strains of this species and all of them showed identical patterns.

The genus Pichia, in the description given by Kurtzman (1984) is, with 57 species, the largest ascomycetous yeast genus. A wide variation exists in their morphological and physiological responses, and some have been transferred to the genera Issatchenkia and Debaryomyces (Krejer-van Rij, 1987). In this study we analysed 20 species recovered from diverse habitats (insects, soils and drinks). The complexity of this genus is reflected in the heterogeneity in the sizes of their PCR fragment lengths, ranging from $380 \mathrm{bp}$ in Pichia pastoris (CECT 11078) to 750 in Pichia angusta (CECT 10220). We obtained unique species patterns with the exception of the pair Pichia segobiensis (CECT 10210) and Pichia stipitis (CECT 1922), which were impossible to differentiate with all the endonucleases tested $(A l u \mathrm{I}$, CfoI, DdeI, HaeIII, HinfI, ScrFI and TaqI). We paid special attention to the species isolated mainly from fruit, soft drinks and alcoholic beverages, such as 
Table 1. Size in bp of the PCR products and the restriction fragments of teleomorphic and anamorphic ascomycetous yeasts

\begin{tabular}{|c|c|c|c|c|c|}
\hline \multirow[t]{2}{*}{ Species* } & \multirow[t]{2}{*}{ CECT strain no. } & \multirow[t]{2}{*}{$\mathbf{A P} \dagger$} & \multicolumn{3}{|c|}{ Restriction fragments } \\
\hline & & & Cfol & HaeIII & HinfI \\
\hline Candida agrestis & $11179^{\mathrm{T}}$ & 400 & $210+190$ & $290+110$ & $220+180$ \\
\hline Candida albicans & $1002^{\mathrm{T}}, 1392,1439,11181,11182$ & 550 & $290+260$ & $460+90$ & $280+270$ \\
\hline Candida apicola & 11167 & 750 & $340+310+100$ & 730 & $390+195+160$ \\
\hline Candida auringiensis & $10623^{\mathrm{T}}$ & 450 & $240+210$ & 440 & $340+100$ \\
\hline Candida beechii & $11165^{\mathrm{T}}$ & 650 & $300+300$ & $400+140+90$ & $330+330$ \\
\hline Candida boidinii & $1014^{\mathrm{T}}, 11153$ & 750 & $350+310+90$ & 700 & $390+190+160$ \\
\hline Candida cacaoi & $11059^{\mathrm{T}}$ & 700 & $310+310+80$ & $430+180+90$ & $360+175+165$ \\
\hline Candida cantarellii & 11170 & 700 & $300+300+85$ & $435+180+90$ & $360+175+165$ \\
\hline Candida diversa & $11159,11178^{\mathrm{T}}$ & 450 & $160+100+100+90$ & 410 & $220+200$ \\
\hline Candida ergatensis & $10261^{\mathrm{T}}$ & 650 & $300+275$ & $500+150$ & $325+190+130$ \\
\hline Candida glabrata & 1448 & 800 & 395 & 675 & $360+270$ \\
\hline Candida incommunis & $11166^{\mathrm{T}}$ & 425 & 400 & 425 & $225+200$ \\
\hline Candida intermedia var. intermedia & $10118,11054,11154$ & 425 & $220+190$ & 410 & $225+200$ \\
\hline Candida magnoliae & 10664 & 425 & $200+190$ & $285+140$ & $225+200$ \\
\hline Candida maltosa & $1936^{\mathrm{T}}$ & 550 & $300+250$ & 550 & $280+270$ \\
\hline Candida mesenterica & 1025 & 650 & 600 & $500+150$ & $320+220+100$ \\
\hline Candida montana & $11180^{\mathrm{T}}$ & 525 & $250+230$ & $400+125$ & $270+250$ \\
\hline Candida norvegica & 10310 & 580 & 510 & $370+190$ & $290+260$ \\
\hline Candida parapsilosis & $1449,10434,10437$ & 550 & $300+240$ & $400+115$ & $290+260$ \\
\hline Candida sake & $1044,10034,10276$ & 450 & $250+200$ & 450 & $230+220$ \\
\hline Candida salmanticensis & $10487^{\mathrm{T}}$ & 450 & $240+200$ & 440 & 410 \\
\hline Candida sorbosa & 11204 & 500 & $215+100+80$ & $300+90+90$ & $270+120+110$ \\
\hline Candida stellata & $11046,11109,11110$ & 475 & $215+110+80+60$ & 475 & $235+235$ \\
\hline Candida terebra & 11015 & 650 & $300+250+90$ & $400+150+100$ & $325+325$ \\
\hline Candida tropicalis & 1440 & 550 & $280+250$ & $450+90$ & $270+270$ \\
\hline Candida wandervaltii & 11169 & 480 & $225+150+100$ & 480 & $240+240$ \\
\hline Candida vinaria & $11177^{\mathrm{T}}$ & 560 & $290+160+110$ & $460+100$ & $250+200+100$ \\
\hline Candida vini & $10053,11137,11174$ & 500 & $230+180+90$ & 500 & $220+220+60$ \\
\hline Candida wickerhamii & $10231^{\mathrm{T}}$ & 660 & 600 & $560+100$ & $325+325$ \\
\hline Candida zeylanoides & 1441 & 620 & $295+295$ & $415+140$ & $310+310$ \\
\hline Debaromyces hansenii & 10386 & 650 & $300+300+50$ & $420+150+90$ & $325+325$ \\
\hline \multirow[t]{2}{*}{ Deharomyces polymorphus } & 1132 & 730 & $300+200+180+100$ & $650+80$ & $310+200+140+100$ \\
\hline & 10043 & 650 & $300+300+50$ & $420+150+90$ & $325+325$ \\
\hline Debaromyces pseudopolymorphus & 10018,10286 & 650 & $300+300+50$ & $420+150+90$ & $325+325$ \\
\hline Dekkera anomala & $11162^{\mathrm{T}}$ & 800 & $340+340+120$ & 800 & $360+190+160+80$ \\
\hline Dekkera bruxellensis & $1009,1010,1451^{\mathrm{T}}, 1452,11045$ & 485 & $255+140+90$ & $375+95$ & $270+215$ \\
\hline Hansenula mrakii & 11031 & 610 & 580 & $330+140+85$ & $305+305$ \\
\hline Issatchenkia terricola & 11139,11176 & 450 & $130+100+90+85+45$ & $290+125$ & $240+105+105$ \\
\hline Kluyveromyces aestuarii & 1949,1955 & 750 & $285+165+125+100+65$ & $640+95$ & $365+180+165+40$ \\
\hline Kluyveromyces africanus & $1963^{\mathrm{T}}$ & 780 & $350+320+105$ & $680+110$ & $395+385$ \\
\hline Kluyveromyces bacillisporus & $1979^{\mathrm{T}}$ & 820 & $330+305+105+105$ & $550+280$ & $310+285+115+110$ \\
\hline Kluyveromyces blattae & $1964^{\mathrm{T}}$ & 530 & $280+250$ & $380+150$ & $295+235$ \\
\hline Kluyveromyces delphensis & $1954^{T}$ & 765 & $350+320+90$ & $420+240+100$ & $380+245+140$ \\
\hline Kluyveromyces dobzhanskii & $1952^{\mathrm{T}}, 10147,10177,10194$ & 740 & $285+190+165+90$ & $655+80$ & $240+185+120+115+80$ \\
\hline Kluyveromyces lactis & $1121,1961^{\mathrm{NT}}, 10356,10669$ & 740 & $285+190+165+90$ & $655+80$ & $290+180+120+80+65$ \\
\hline $\begin{array}{l}\text { (Kluyveromyces marxianus var. } \\
\text { drosophilarum) }\end{array}$ & 10390,11337 & 740 & $285+190+165+90$ & $655+80$ & $240+185+120+80+65+50$ \\
\hline $\begin{array}{l}\text { (Kluyveromyces marxianus var. } \\
\text { phaseolosporus) }\end{array}$ & 11340 & 740 & $285+190+165+90$ & $655+80$ & $240+185+185+65+50+15$ \\
\hline Kluyveromyces lodderae & $1126^{\mathrm{T}}$ & 740 & $320+320+100$ & $640+100$ & $350+240+110+55$ \\
\hline Kluyveromyces marxianus & $1123,10368,10585^{T}, 10668$ & 740 & $285+185+140+100$ & $655+80$ & $240+185+120+80+65+50$ \\
\hline Kluyveromyces phaffii & $10646^{\mathrm{T}}$ & 575 & $190+150+100+95+60$ & $520+50$ & $315+250$ \\
\hline Kluyveromyces polysporus & $1960^{\mathrm{T}}$ & 755 & $330+320+110$ & $650+100$ & $390+365$ \\
\hline Kluyveromyces thermotolerans & $1962,10141,10183,10559$ & 700 & $315+285+95$ & $310+215+90+90$ & $355+345$ \\
\hline Khyveromyces waltii & $1950^{\mathrm{T}}$ & 700 & $315+285+95$ & $310+215+90+90$ & $350+350$ \\
\hline Kluyveromyces wickerhamii & $1953,1966^{\mathrm{T}}$ & 740 & $285+190+165+100$ & $665+80$ & $240+185+120+80+65+50$ \\
\hline Kluyveromyces yarrowii & $1958^{\mathrm{T}}$ & 780 & $320+240+110+100$ & $680+100$ & $410+370$ \\
\hline Lodderomyces elongisporus & 10645 & 600 & $330+250$ & 550 & $300+270$ \\
\hline Metschnikowia pulcherrima & $1691,10408,10546,11202^{\mathrm{T}}$ & 400 & $205+100+95$ & $280+100$ & $200+190$ \\
\hline Pichia angophorae & $1899^{\mathrm{T}}$ & 750 & $350+320+80$ & 750 & $410+340$ \\
\hline Pichia angusta & 10220 & 750 & $370+300+80$ & $500+150+100$ & $425+315$ \\
\hline Pichia anomala & $1110,1114,10320,10410,10590,10591,10667$ & 650 & 575 & $600+50$ & $310+310$ \\
\hline Pichia capsulata & 11013 & 650 & $290+290$ & $490+125+35$ & $230+175+150+95$ \\
\hline Pichia carsonii & $10227^{\mathrm{T}}, 10230,10541$ & 650 & $290+290$ & $410+145+90$ & $310+310$ \\
\hline Pichia castillae & $10017^{\mathrm{T}}$ & 650 & $300+290+60$ & $410+145+90$ & $310+175+140$ \\
\hline Pichia delftensis & $10238^{\mathrm{T}}$ & 500 & $260+180+60$ & 500 & $270+230$ \\
\hline Pichia fabianii & 1921 & 650 & $290+200+90$ & $560+90$ & $310+310$ \\
\hline Pichia farinosa & 1447,10348 & 700 & $300+300+80$ & $440+175+80$ & $350+185+160$ \\
\hline Pichia fermentans & $1455^{\mathrm{T}}, 10064,10078,10413,10454$ & 450 & $170+100+100+80$ & $340+80+30$ & $250+200$ \\
\hline Pichia fluxuum & 10659 & 500 & $230+180+90$ & 500 & $240+240$ \\
\hline
\end{tabular}


Table 1 (cont.)

\begin{tabular}{|c|c|c|c|c|c|}
\hline \multirow[t]{2}{*}{ Species* } & \multirow[t]{2}{*}{ CECT strain no. } & \multirow[t]{2}{*}{$\mathbf{A P}^{\dagger}$} & \multicolumn{3}{|c|}{ Restriction fragments } \\
\hline & & & Cfol & HaeIII & Hinfl \\
\hline Pichia guilliermondii & $1019,1021,1438,1456^{\mathrm{T}}$ & 625 & $300+265+60$ & $400+115+90$ & $320+300$ \\
\hline Pichia jadinii & 1060 & 575 & $280+230$ & $390+145+40$ & $300+275$ \\
\hline Pichia kluyveri & $11023^{\mathrm{T}}$ & 450 & $175+115+80+80$ & $370+80$ & $250+200$ \\
\hline \multirow[t]{2}{*}{ Pichia membranaefaciens } & $1115,10570,10568$ & 500 & $175+110+90+75$ & $330+90+50$ & $275+200$ \\
\hline & 10037,10113 & 500 & $260+110+75$ & $330+90+50$ & $275+200$ \\
\hline Pichia pastoris & 11078 & 380 & 360 & 380 & $250+130$ \\
\hline Pichia pijperi & $10662^{\mathrm{T}}$ & 650 & $300+300$ & $425+140+85$ & $310+190+120$ \\
\hline Pichia scolyti & 10661 & 650 & $300+300$ & $425+140+85$ & $310+310$ \\
\hline Pichia segobiensis & $10210^{\mathrm{T}}$ & 650 & $300+285$ & $490+140$ & $310+310$ \\
\hline Pichia stipitis & $1922^{\mathrm{T}}$ & 650 & $300+285$ & $490+140$ & $310+310$ \\
\hline Saccharomyces bayanus & $1941^{\mathrm{T}}, 1969$ & 880 & $385+365$ & $500+220+145$ & $365+155$ \\
\hline Saccharomyces cerevisiae & $1942^{\mathrm{T}}, 1971$ & 880 & $385+365$ & $320+230+180+150$ & $365+155$ \\
\hline Saccharomyces exiguus & $11192^{\mathrm{T}}$ & 675 & $320+200+90$ & $400+200+75$ & $400+275$ \\
\hline Saccharomyces paradoxus & $1939^{\mathrm{T}}, 11143$ & 880 & $385+365$ & $320+230+180+150$ & $440+440$ \\
\hline Saccharomyces pastorianus & $1940^{\mathrm{T}}, 1320$ & 880 & $385+365$ & $500+220+145$ & $365+155$ \\
\hline Saccharomycodes ludwigii & $10450^{\mathrm{T}}, 11141,11191$ & 750 & $360+350$ & 700 & $450+275$ \\
\hline Saccharomycopsis capsularis & $10653^{\mathrm{NT}}$ & 675 & 600 & $500+95+80$ & $335+335$ \\
\hline \multirow{2}{*}{$\begin{array}{l}\text { Schizosaccharomyces pombe var. } \\
\text { pombe }\end{array}$} & 1379.11197 & 1050 & $600+400$ & 1050 & $600+450$ \\
\hline & 1378,10685 & 950 & $525+375$ & 950 & $500+425$ \\
\hline Torulaspora delbrueckii & $\begin{array}{l}1880,10558,10589,10651,10676,10683 \\
10693,10694,11146,11199\end{array}$ & 800 & $330+220+150+100$ & 800 & $410+380$ \\
\hline Torulaspora globosa & 10655 & 650 & $300+300$ & $425+150+90$ & $325+325$ \\
\hline Torulaspora pretoriensis & 10679,10680 & 825 & $375+330+110$ & 800 & $380+290+125$ \\
\hline Wickerhamiella domerquiae & $11173^{\mathrm{T}}$ & 825 & $300+250$ & 525 & $275+275$ \\
\hline Yarrowia lipolytica & 1240 & 380 & $210+170$ & 380 & $190+190$ \\
\hline Zygoascus hellenicus & 11163 & 650 & $325+325$ & 625 & $350+170+130$ \\
\hline Zygosaccharomyces bailii & $10674,11042,11043$ & 790 & $320+270+95+95$ & $690+90$ & $340+225+160+55$ \\
\hline Zygosaccharomyces bisporus & $11055^{\mathrm{T}}$ & 790 & $300+275+110+90$ & $690+100$ & $390+225+150$ \\
\hline Zygosaccharomyces cidri & 10657 & 700 & $310+280+90$ & $300+210+95+95$ & $340+340$ \\
\hline Zygosaccharomyces fermentati & $10382,10478,11056^{\mathrm{T}}$ & 700 & $310+280+90$ & $300+210+95+95$ & $340+340$ \\
\hline Zygosaccharomyces florentinus & $11200^{\mathrm{T}}$ & 600 & $275+185+80$ & 590 & $295+295$ \\
\hline Zygosaccharomyces mellis & $11057^{\mathrm{LTT}}$ & 850 & $350+250+210$ & $560+200+90$ & $400+270+180$ \\
\hline \multirow[t]{2}{*}{ Zygosaccharomyces microellipsoides } & $11198^{\mathrm{T}}$ & 825 & $350+285+100+90$ & $725+100$ & $425+400$ \\
\hline & 10039 & 825 & $330+220+150+100$ & 800 & $425+400$ \\
\hline Zygosaccharomyces mrakii & $10656^{\mathrm{T}}$ & 650 & $290+280+80$ & $390+115+80$ & $300+190+130$ \\
\hline Zygosaccharomyces rouxii & $1230,1232,10137,11136,11189$ & 750 & $290+200+170+90$ & $400+210+90$ & $350+260+140$ \\
\hline
\end{tabular}

* Original epithets are indicated in parentheses.

$\dagger$ PCR-amplified product.

Table 2. Size in bp of PCR products and restriction fragments of ascomycetous yeasts in the genus Hanseniaspora

To differentiate these species, restriction analysis with $D d e I$ was required.

\begin{tabular}{|c|c|c|c|c|c|c|}
\hline \multirow[t]{2}{*}{ Species } & \multirow[t]{2}{*}{ CECT strain no. } & \multirow[t]{2}{*}{$\mathbf{A P}^{*}$} & \multicolumn{4}{|c|}{ Restriction fragments } \\
\hline & & & CfoI & DdeI & HaelII & Hinf1 \\
\hline $\begin{array}{l}\text { Hanseniaspora } \\
\text { guilliermondii }\end{array}$ & $\begin{array}{l}11027,11029^{\mathrm{T}} \\
11102,11104 \\
11111,11155 \\
11205\end{array}$ & 750 & $320+310+105$ & $380+180+95+80$ & 750 & $350+200+180$ \\
\hline Hanseniaspora osmophila & $\begin{array}{l}11206 \\
11207\end{array}$ & $\begin{array}{l}750 \\
750\end{array}$ & $\begin{array}{l}275+150+135+95+75 \\
275+185+150+95\end{array}$ & $\begin{array}{l}650+100 \\
650+100\end{array}$ & $\begin{array}{l}460+120+90+80 \\
460+120+90+80\end{array}$ & $\begin{array}{l}390+360 \\
390+360\end{array}$ \\
\hline Hanseniaspora uvarum & $\begin{array}{l}1444,10389 \\
11105,11106 \\
11107,11156 \\
11172\end{array}$ & 750 & $320+310+105$ & $300+180+95+90+85$ & 750 & $350+200+180$ \\
\hline Hanseniaspora valbyensis & $1445^{\mathrm{T}}, 10122$ & 750 & $630+120$ & $290+150+95+95$ & 750 & $250+220+170+105$ \\
\hline
\end{tabular}

* PCR-amplified product. 
Table 3. Size in bp of PCR products and restriction fragments of teleomorphic and anamorphic basidiomycetous yeasts

\begin{tabular}{|c|c|c|c|c|c|}
\hline \multirow[t]{2}{*}{ Species* } & \multirow[t]{2}{*}{ CECT strain no. } & \multirow[t]{2}{*}{$\mathbf{A P} \dagger$} & \multicolumn{3}{|c|}{ Restriction fragments } \\
\hline & & & CfoI & HaeIII & HinfI \\
\hline Cryptococcus albidus & $1069,1072,1073$ & 630 & $330+300$ & $500+70+60$ & $350+160+120$ \\
\hline $\begin{array}{l}\text { (Cryptococcus diffluens var. } \\
\text { uruguaiensis) }\end{array}$ & $1086^{\mathrm{T}}, 11138$ & 630 & $330+300$ & $500+70+60$ & $350+280$ \\
\hline Cryptococcus ater & $1084^{\mathrm{T}}$ & 650 & $350+300$ & $500+90+60$ & $275+245+130$ \\
\hline Cryptococcus bhutanensis & $1085^{\mathrm{T}}$ & 630 & $330+300$ & $500+70+60$ & $350+280$ \\
\hline Cryptococcus curvatus & 1992 & 700 & $305+300+50+45$ & $460+160+80$ & $320+320+60$ \\
\hline Cryptococcus dimennae & $1087^{\mathrm{T}}$ & 500 & $255+245$ & $420+40+40$ & $280+220$ \\
\hline Cryptococcus flavus & 1074 & 540 & $190+180+90$ & $460+80$ & $230+170+70+60$ \\
\hline Cryptococcus gastricus & $1089^{\mathrm{T}}$ & 630 & 630 & $505+65+60$ & $235+150+135+110$ \\
\hline Cryptococcus himalayensis & $1090^{\mathrm{T}}$ & 660 & $345+315$ & $400+70+70+60+60$ & $370+290$ \\
\hline Cryptococcus humicola & 1251 & 520 & $265+255$ & $425+55+40$ & $230+230+60$ \\
\hline Cryptococcus hungaricus & 1091 & 500 & $260+240$ & 500 & $260+240$ \\
\hline Cryptococcus kuetzingii & $1093^{\mathrm{T}}$ & 630 & $330+300$ & $500+70+60$ & $350+280$ \\
\hline \multirow[t]{2}{*}{ Cryptococcus laurentii } & 1095 & 600 & 575 & 600 & $340+260$ \\
\hline & 11171 & 550 & $290+260$ & $375+100$ & $270+270$ \\
\hline Cryptococcus luteolus & 1099 & 490 & $260+230$ & 490 & $250+240$ \\
\hline Cryptococcus macerans & $1101^{\mathrm{T}}$ & 650 & $260+200+95+95$ & $440+80+70+60$ & $270+200+175$ \\
\hline Cryptococcus magnus & 1103 & 650 & $355+295$ & $495+95+60$ & $270+240+140$ \\
\hline Cryptococcus marinus & 1975 & 500 & $260+140+90$ & 500 & $220+210+70$ \\
\hline Cryptococcus skinneri & $1105^{\mathrm{T}}$ & 565 & $260+200$ & $400+110$ & $220+150+120+60$ \\
\hline Cryptococcus terreus & $1106^{\mathrm{T}}$ & 900 & $400+180+165+155$ & $670+230$ & $360+270+270$ \\
\hline Cryptococcus uniguttulatus & 1108 & 650 & $355+295$ & $500+150$ & $270+240+140$ \\
\hline Filobasidium capsuligenum & 11190 & 650 & $340+300$ & $550+100$ & $360+280$ \\
\hline Rhodotorula acuta & 11175 & 675 & $355+315$ & 650 & $250+190+185$ \\
\hline Rhodotorula glutinis & 1137 & 640 & $320+240+80$ & $430+210$ & $340+225+75$ \\
\hline Rhodotorula graminis & 10097 & 660 & $320+290$ & $400+215$ & $230+215+150$ \\
\hline Rhodotorula minuta & 10033 & 660 & $300+300$ & $400+215$ & $345+215$ \\
\hline Rhodotorula mucilaginosa & 11010 & 640 & $320+240+80$ & $425+215$ & $340+225+75$ \\
\hline Sporidiobolus pararoseus & 10153,1270 & 630 & $320+310$ & $410+220$ & $250+230+120$ \\
\hline Sporidiobolus salmonicolor & 1274,11115 & 600 & $400+240$ & $410+140+95$ & $300+250+125$ \\
\hline Sporobolomyces roseus & 1930 & 610 & $320+250$ & 600 & $280+120+105+105$ \\
\hline Trichosporon beigelii & 1304 & 550 & $280+90+90+90$ & 525 & $300+250$ \\
\hline
\end{tabular}

* Original epithets are indicated in parentheses.

$\dagger$ PCR-amplified product.

Pichia anomala, Pichia fermentans, Pichia guilliermondii and Pichia membranaefaciens. Among the strains of Pichia membranaefaciens we found two different patterns with CfoI (CECT 10037 and CECT 10113). This could be a way recognizing varieties within this heterogeneous species.

Schizosaccharomyces pombe, characterized by its special mode of vegetative reproduction and a certain degree of osmophily, is probably the only one to cause food spoilage of the three species of the genus. This species presents a great variability in the size of the $5.8 \mathrm{~S}$ region, as described by Schaak et al. (1982). In the four strains analysed, we found two fragment lengths (950 bp and $1050 \mathrm{bp}$ ) that agree with the two different - restriction patterns, one of them for the strains CECT
1378 and CECT 10685, and the other for CECT 1379 and CECT 11197 (see Table 1).

In the genus Torulaspora three species are accepted according to Barnett et al. (1990), although the most frequently isolated from foods is Torulaspora delbrueckii. We observed the same pattern for ten strains of this species, and species-specific patterns for the other two species of the genus, Torulaspora globosa and Torulaspora pretoriensis.

The species of the genus Zygosaccharomyces are important osmotolerant food spoilage ascomycetous yeasts. In this study, we analysed 18 strains from the 9 species of this genus, and we could associate each species with a particular pattern, with the exception of 
Zygosaccharomyces cidri and Zygosaccharomyces fermentati, which yielded the same pattern with all the endonucleases used (AluI, CfoI, Dde I, HaeIII, HinfI, ScrFI and TaqI). The two strains of Zygosaccharomyces microellipsoides showed the same pattern using only the endonuclease HinfI, but were polymorphic for the other two enzymes. For this reason we recommend the use of only HinfI to identify this species.

\section{Teleomorphic and anamorphic basidiomycetous yeasts}

Species belonging to the genus Cryptococcus are defined by a combination of morphological and physiological characters, the absence of pseudomycelium, colony pigmentation and ultrastructure of the septal pore (Guého et al., 1989). Due to the lack of fermentation and sexual reproduction their identification by conventional approaches is rather difficult, so we propose this new method also for the identification of the species of this genus. The amplified fragments present a high degree of variation in size, ranging from $490 \mathrm{bp}$ for Cryptococcus luteolus (CECT 1099) to $900 \mathrm{bp}$ for Cryptococcus terreus (CECT 1106). Using the endonucleases $H a e I I I$ and $C f o I$, all the strains of Cryptococcus albidus var. albidus yielded the same restriction fragments, but with HinfI the strains CECT 11138 and CECT 1086 (type strain of Cryptococcus diffluens var. uruguaiensis) showed a different restriction pattern, which could be an indication of the existence of a different variety or species. The species Cryptococcus magnus and Cryptococcus uniguttulatus have the same PCR product size and restriction patterns with HinfI and $C f o$ I. They can be distinguished by using the endonuclease HaeIII (see Table 3).

The genera Rhodotorula, Sporidiobolus and Sporobolomyces include basidiomycetous yeasts producing red- or orange-pigmented colonies. An important characteristic of Rhodotorula species is the inability to assimilate inositol, which distinguishes Rhodotorula from Cryptococcus (Krejer-van Rij, 1987). We could differentiate the five species analysed in the present study. The species Rhodotorula graminis and Rhodotorula minuta had the same pattern with HaeIII, but different patterns with $C f o \mathrm{I}$ and $H$ infl. With these endonucleases it is possible to obtain a species-specific pattern.

\section{Conclusions}

The restriction patterns of the 5.8S-ITS region have been used to differentiate 132 yeast species belonging to 25 different yeast genera, the majority isolated from food and some additional species representing related genera. Analysis of Tables 1, 2 and 3 confirmed that this method can be used to differentiate all the species with the exceptions of $\mathrm{Cr}$. albidus (strains previously identified as $\mathrm{Cr}$. diffluens var. uruguaiensis)/Cr.
bhutanensis/Cr. kuetzingii; Deb. polymorphus/Deb. pseudopolymorphus; P. segobiensis/P. stipitis; Sacch. bayanus/Sacch. pastorianus; Zygosacch. cidri/ $Z y$ gosacch.fermentati. In all cases, the method proved to be reproducible and very useful to easily and rapidly identify and classify all the species included in the present work. This special feature has been used for identification of wine yeast species (Guillamón et al., 1998) and also for strains isolated from cheese and fermented sausages (unpublished).

Using this method, the anamorph and teleomorph forms yielded the same pattern, as exemplified by the pairs $B r$. bruxellensis/D. bruxellensis, $H^{\prime}$ spora uvarum $/ \mathrm{Kl}$. apiculata and T'spora delbruekii/C. colliculosa. In addition, the majority of the strains of the same species showed the same restriction pattern with all the enzymes analysed, with the exception of $K$. lactis (Belloch et al., 1998) and Cr. albidus, where strains previously identified as synonymous could be recognized as varieties.

Finally, the results presented in this work constitute an initial database, and should be supplemented with further species not used in the present work. To prevent errors we recommend the use of the same conditions that we have applied in this study, namely to separate the PCR products on $1.4 \%$ and the restriction fragments on $3 \%$ agarose gels. In the case of doubt, we suggest including the type strains in the same gel and, finally, it is worth noting that fragments smaller than $50 \mathrm{bp}$ could not be clearly visualized and were not considered.

\section{ACKNOWLEDGEMENTS}

This work was supported by CICYT grants to A. Q. (ALI960457-CO2-01) and to F.U. (ALI96-0457-CO2-02). B.E. was the recipient a FPI fellowship from the Spanish Government. We express our gratitude to J.M. Guillamón for participating in the application of this method to wine yeast identification. These preliminary results showed us the potentiality of the technique. Thanks are due to $\mathrm{D}$. Ramón and E. Barrio for the critical discussion of the manuscript.

\section{REFERENCES}

Augustyn, O. P. H., Ferreira, D. \& Kock, J. L. F. (1991). Differentiation between yeast species, and strains within species by cellular fatty acid analysis. 4. Saccharomyces sensu stricto, Hanseniaspora, Saccharomycodes and Wickerhamiella. Syst Appl Microbiol 14, 324-334.

Baleiras Couto, M. M., van der Vossen, J. M., Hofstra, H. \& in 't Veld, J. H. (1994). RAPD analysis: a rapid technique for differentiation of spoilage yeasts. Int $J$ Food Microbiol 24, 249-260.

Baleiras Couto, M. M., Eijsma, B., Hosfra, H., in 't Veld, J. H. \& van der Vossen, J. M. (1996). Evaluation of molecular typing techniques to assign genetic diversity among Saccharomyces cerevisiae strains. Appl Environ Microbiol 62, 41-46.

Barnett, J. A., Payne, R. W. \& Yarrow, D. (1990). Yeasts: Characteristics and Identification, 2nd edn. Cambridge: Cambridge University Press. 
Belloch, C., Barrio, E., Uruburu, F., García, M. D. \& Querol, A. (1997). Characterization of four species of the genus Kluyveromyces by mitochondrial DNA restriction analysis. Syst Appl Microbiol 20, 397-408.

Belloch, C., Barrio, E., García, M. D. \& Querol, A. (1998). Phylogenetic reconstruction of the genus Kluyveromyces: restriction map analysis of the 5.8S rRNA gene and the two ribosomal internal transcribed spacers. Syst Appl Microbiol 21, 266-273.

Botelho, A. R. \& Planta, R. J. (1994). Specific identification of Candida albicans by hybridization with oligonucleotides derived from ribosomal DNA internal spacers. Yeast 10, 709-717.

Cai, J., Roberts, I. N. \& Collins, M. D. (1996). Phylogenetic relationships among members of the ascomycetous yeast genera Brettanomyces, Debaryomyces, Dekkera and Kluyveromyces deduced by small-subunit rRNA gene sequences. Int $J$ Syst Bacteriol 46, 542-549.

Carlie, M. J. \& Watkinson, S. C. (1994). The Fungi. London: Academic Press.

Cottrell, M., Kock, J. L. F., Lategan, P. M. \& Britz, T. J. (1986). Long-chain fatty acid composition as an aid in the classification of the genus Saccharomyces. Syst Appl Microbiol 8, 166-168.

Deák, T. (1993). Simplified techniques for identifying foodborne yeasts. Int J Food Microbiol 19, 15-26.

Deák, T. (1995). Methods for the rapid detection and identification of yeasts in foods. Trends Food Sci Tech 6, 287-292.

Deák, T. \& Beuchat, L. R. (1996). Handbook of Food Spoilage Yeasts. Boca Raton, FL: CRC Press.

Golden, D. A., Beuchat, L. R. \& Hitchcock, H. L. (1994). Changes in fatty acid composition of Zygosaccharomyces rouxii as influenced by solutes, potassium sorbate and incubation temperature. Int J Food Microbiol 21, 293-303.

Guého, E., Kurtzman, C. P. \& Peterson, S. W. (1989). Evolutionary affinities of heterobasidiomycetous yeasts estimated from $18 \mathrm{~S}$ and $25 \mathrm{~S}$ ribosomal RNA sequence divergence. Syst Appl Microbiol 12, 230-236.

Guillamón, J. M., Querol, A., Jimenez, M. \& Huerta, T. (1993). Phylogenetic relationships among wine yeast strains based on electrophoretic whole-cell protein patterns. Int J Food Microbiol 18, 115-125.

Guillamón, J. M., Barrio, E., Huerta, T. \& Querol, A. (1994). Rapid characterization of four species of the Saccahromyces sensu stricto complex according to mitochondrial DNA patterns. Int $J$ Syst Bacteriol 44, 708-714.

Guillamón, J. M., Barrio, E. \& Querol, A. (1996). Characterization of wine yeast strains of the Saccharomyces genus on the basis of molecular markers. Relationships between genetic distance and geographic origin. Syst Appl Microbiol 19, 122-132.

Guillamón, J. M., Sanchez, I. \& Huerta, T. (1997). Rapid characterization of wild and collection strains of the genus Zygosaccharomyces according to mitochondrial DNA patterns. FEMS Microbiol Lett 147, 267-272.

Guillamón, J. M., Sabaté, J., Barrio, E., Cano, J. \& Querol, A. (1998). Rapid identification of wine yeast species based on RFLP analysis of the ribosomal ITS regions. Arch Microbiol 169, 387-392.

Huffman, J. L., Molina, F. I. \& Jong, S. C. (1992). Authentication of ATCC strains in the Saccharomyces cerevisiae complex by PCR fingerprinting. Exp Mycol 16, 316-319.

Ibeas, J. I., Lozano, I., Perdigones, L. \& Jimenez, J. (1996). Detection of Dekkera/Brettanomyces strains in sherry by a nested PCR method. Appl Environ Microbiol 62, 998-1003.
Ibeas, J. I., Lozano, I., Perdigones, F. \& Jimenez, J. (1997). Dynamics of flor yeast populations during the biological aging of sherry wines. Am J Enol Vitic 48, 75-79.

Iwaguchi, S.-I., Homma, M. \& Tanaka, K. (1990). Variation in the electrophoretic karyotype analysed by the assignment of DNA probes in Candida albicans. J Gen Microbiol 136, 2433-2442.

James, S. A., Collins, M. D. \& Roberts, I. N. (1996). Use of an rRNA internal transcribed spacer region to distinguish phylogenetically closely related species of the genera Zygosaccharomyces and Torulaspora. Int J Syst Bacteriol 46, 189-194.

Jordan, J. A. (1994). PCR identification of four medically important Candida species by using a single primer pair. J Clin Microbiol 32, 2962-2967.

Krejer-van Rij, N. J. W. (1984). The Yeasts: a Taxonomic Study, 3rd edn. Amsteram: Elsevier.

Krejer-van Rij, N. J. W. (1987). Classification of yeasts. In The Yeasts, vol 1, Biology of the Yeasts, 2nd edn, pp. 5-61. Edited by A. H. Rose \& J. S. Harrison. London: Academic Press.

Kurtzman, C. P. (1984). Synonomy of the yeast genera Hansenula and Pichia demonstrated through comparisons of deoxyribonucleic acid relatedness. Antonie Leeuwenhoek 50, 209-217.

Kurtzman, C. P. (1992). rRNA sequence comparisons for assessing phylogenetic relationships among yeasts. Int $J$ Syst Bacteriol 42, 1-6.

Kurtzman, C. P. (1993). Systematics of the ascomycetous yeasts assessed from ribosomal RNA sequence divergence. Antonie Leeuwenhoek 63, 165-174.

Kurtzman, C. P. \& Fell, J. W. (1998). The Yeasts - a Taxonomic Study, 4th edn. Amsterdam: Elsevier.

Li, W. H. (1997). Molecular Evolution. Sunderland, MA: Sinauer Associates.

Lin, C. C. S. \& Fung, Y. (1987). Conventional and rapid methods for yeast identification. Crit Rev Microbiol 14, 273-289.

Lopandic, K., Prillinger, H., Molnár, O. \& Gimenez-Jurado, G. (1996). Molecular characterization and genotypic identification of Metschnikowia species. Syst Appl Microbiol 19, 393-402.

Meyer, S. A., Smith, M. Th. \& Simione, F. P., Jr (1978). Systematics of Hanseniaspora Zikes and Kloeckera Janke. Antonie Leeuwenhoek J Microbiol Serol 44, 79-96.

Molina, F. I., Inoue, T. \& Jong, S. C. (1992). Ribosomal DNA restriction analysis reveals genetic heterogeneity in Saccharomyces cerevisiae Meyen ex Hansen. Int J Syst Bacteriol 42, 499-502.

Moreira da Silva, M., Malfeito-Ferreira, M., Aubyn, St. \& Loureiro, V. (1994). Long-chain fatty acid composition as a criterion for yeast distinction in the brewing industry. J Inst Brew 100, 17-22.

Nadal, D., Colomer, B. \& Piña, B. (1996). Molecular polymorphism distribution in phenotypically distinct populations of wine yeast strains. Appl Environ Microbiol 62, 1944-1950.

Naumov, G. I., Naumova, E. S. \& Sniegowski, P. D. (1997). Differentiation of European and far east Asian populations of Saccharomyces paradoxus by allozyme analysis. Int $J$ Syst Bacteriol 47, 341-344.

Perez, L., Martinez, P., Codon, A. C. \& Benitez, T. (1995). Physiological and molecular characterization of flor yeasts: polymorphisms of flor yeast populations. Yeast 11, 1399-1411. Querol, A., Barrio, E., Huerta, T. \& Ramón, D. (1992a). Molecular monitoring of wine fermentations conducted by active dry yeast strains. Appl Environ Microbiol 58, 2948-2953.

Querol, A., Barrio, E. \& Ramón, D. (1992b). A comparative study 
of different methods of yeast strain characterization. Syst Appl Microbiol 15, 439-446.

Quesada, M. P. \& Cenis, J. L. (1995). Use of random amplified polymorphic DNA (RAPD)-PCR in the characterization of wine yeasts. Am J Enol Vitic 46, 204-208.

Romano, A., Casaregola, S., Torre, P. \& Gaillardin, C. (1996). Use of RAPD and mitochondrial DNA RFLP for typing of Candida zeylanoides and Debaryomyces hansenii yeast strains isolated from cheese. Syst Appl Microbiol 19, 255-264.

Schaak, J., Mao, J. I. \& Soell, D. G. (1982). The 5.8S RNA gene sequence and the ribosomal repeat of Schizosaccharomyces pombe. Nucleic Acids Res 10, 2851-2864.

Schüctz, M. \& Gafner, J. (1993). Analysis of yeast diversity during spontaneous and induced alcoholic fermentations. $J \mathrm{Appl}$ Bacteriol 75, 551-558.

Török, T., Rockhold, D. \& King, A. D. (1993). Use of electrophoretic karyotyping and DNA-DNA hybridization in yeast identification. Int $J$ Food Microbiol 19, 63-80.

Tredoux, H. G., Kock, J. L. F., Lategan, P. M. \& Muller, H. B. (1987). A rapid identification technique to differentiate between Saccharomyces cerevisiae strains and other yeasts species in the wine industry. Am J Enol Vitic 38, 161-164.

Valente, P., Gouveia, F. C., de Lemos, G. A., Pimentel, D., van Elsas, J. D., Mendoņa-Hagler, L. C. \& Hagler, A. N. (1996). PCR amplification of the rDNA internal transcribed spacer region for differentiation of Saccharomyces cultures. FEMS Microbiol Lett 137, 253-256.

Vancanneyt, B. P., Hennebert, G. \& Kersters, K. (1991). Differen- tiation of yeast species based on electrophoretic whole-cell protein patterns. Syst Appl Microbiol 14, 23-32.

Vaughan Martini, A. \& Martini, A. (1985). Deoxyribonucleic acid relatedness among species of the genus Saccharomyces sensu stricto. Int J Syst Bacteriol 35, 508-511.

Vaughan Martini, A. \& Martini, A. (1987). Taxonomic revision of the yeast genus Kluyveromyces by nuclear deoxyribonucleic acid reassociation. Int $J$ Syst Bacteriol 37, 380-385.

Versavaud, A. \& Hallet, J. N. (1995). Pulsed-field gel electrophoresis combined with rare-cutting endonucleases for strain identification of Candida famata, Kloeckera apiculata and Schizosaccharomyces pombe with chromosome number and size estimation of the two former. Syst Appl Microbiol 18, 303-309.

van Vuuren, H. J. J. \& van der Meer, L. (1987). Fingerprinting of yeasts by protein electrophoresis. Am J Enol Vitic 38, 49-53.

White, T. J., Bruns, T., Lee, S. \& Taylor, J. (1990). PCR protocols. A guide to methods and applications. In Amplification and Direct Sequencing of Fungal Ribosomal RNA Genes for Phylogenetics, pp. 315-322. Edited by M. A. Innis, D. H. Gelfand, J. J. Sninsky \& T. J. White. San Diego: Academic Press.

Wyder, M. T. \& Puhan, Z. (1997). A rapid method for identification of yeasts from kefyr at species level. Michwissenchaft 52, 327-330.

Yamada, Y., Maeda, K. \& Banno, I. (1992). The phylogenetic relationships of the Q6-equipped species in the teleomorphic apiculate yeast genera Hanseniaspora, Nadsonia and Saccharomycodes based on the partial sequences of $18 \mathrm{~S}$ and $26 \mathrm{~S}$ ribosomal ribonucleic acids. J Gen Appl Microbiol 38, 585-596. 